\title{
L'université et les pouvoirs de la langue
}

Réarticuler l'ordre politique et linguistique en Roumanie au sortir du communisme

The university and the power of language in Romania: reshaping the political and linguistic orders in 1989-1990

\section{Antonela Capelle-Pogăcean}

\section{(2) OpenEdition}

Journals

Édition électronique

URL : http://journals.openedition.org/conflits/18024

DOI : $10.4000 /$ conflits. 18024

ISSN : $1777-5345$

Éditeur :

CCLS - Centre d'études sur les conflits lilberté et sécurité, L'Harmattan

Édition imprimée

Date de publication : 26 décembre 2010

Pagination : 55-72

ISBN : 978-2-296-56047-5

ISSN : 1157-996X

Référence électronique

Antonela Capelle-Pogăcean, «L'université et les pouvoirs de la langue », Cultures \& Conflits [En ligne], 79-80 | Automne/Hiver 2010, mis en ligne le 26 mai 2012, consulté le 30 mars 2021. URL : http:// journals.openedition.org/conflits/18024; DOI : https://doi.org/10.4000/conflits.18024 


\title{
L'université et les pouvoirs de la langue
}

\author{
Réarticuler l'ordre politique et linguistique en Roumanie \\ au sortir du communisme *
}

\section{Antonela CAPELLE-POGĂCEAN}

Antonela Capelle-Pogàcean est chargée de recherche au Centre d'études et de recherches internationales (SciencesPo-CERI) et enseigne à SciencesPo. Ses recherches actuelles portent sur les politiques de l'identité, les recompositions $d u$ religieux et des imaginaires sociaux en Hongrie et Roumanie et l'bistoire sociale du communisme. Elle a notamment publié Vie quotidienne et pouvoir sous le communisme. Consommer à l'Est (co-dirigé avec Nadège Ragaru), Paris, Karthala E CERI, 2010 et Religion(s) et identité(s) en Europe. L'épreuve du pluriel (co-dirigé avec Patrick Michel et Enzo Pace), Paris, Presses de Sciences Po, 2007 ; "Relire Albert Wass en Hongrie et en Roumanie. La construction d'un emblème identitaire » in Denis-Constant Martin (dir.), L'identité en jeux : pouvoirs, identifications, mobilisation, Paris : Karthala E CERI, 2010 ; "L'ethnicité an quotidien : présences et intermittences", East Central Europe, 36 (1), 2009, pp.147-154.

$\mathrm{E}$ n 1989, l'une des premières revendications magyares ${ }^{1}$ qui surgit dans un espace public en proie à des bouleversements spectaculaires au moment de la chute du régime communiste roumain, le 22 décembre, eut pour objet la langue de l'université. À Cluj-Napoca/Kolozsvár, centre urbain de premier ordre en Roumanie avec ses quelque 300000 habitants, capitale historique de la province de Transylvanie disputée depuis le XIXe siècle par les nationalismes hongrois et roumain, des universitaires et des personnalités culturelles magyares s'avançant comme porte-parole de leur communauté, inscrivirent parmi les desideratas collectifs le rétablissement de l'université de langue hongroise. Avec intermittences et sous différentes formes, un tel établissement d'enseignement supérieur avait existé dans la ville depuis 1872. En 1959, l'Université de langue hongroise Bolyai avait fusionné sur décision des autori-

*. Certains accents hongrois et roumains n'ont pas pu être reproduits pour des raisons techniques.

1. Si le français utilise plutôt le terme "magyar » pour désigner les populations hungarophones des États voisins de la Hongrie et « hongrois » pour les citoyens de la Hongrie, la langue hongroise n'opère pas cette distinction. Dans ce texte nous utiliserons d'une manière indifférenciée « hongrois » et « magyar ». 
tés communistes avec son équivalent roumain pour donner naissance à l'Université Babes-Bolyai, l'une des plus prestigieuses du pays 2. Son nom, hommage à deux savants roumain et hongrois ${ }^{3}$, rappelait le caractère bilingue de l'établissement de Cluj/Kolozsvár. Or, l'espace du hongrois s'y était progressivement réduit dans les dernières décennies de la période communiste.

La réouverture de l'Université Bolyai fut promue à l'agenda de l'Union démocratique des Magyars de Roumanie (UDMR) dès l'émergence, le 25 décembre 1989, de cette nouvelle organisation qui revendiqua la représentation politique du groupe hongrois ${ }^{4}$. Plusieurs autres associations ethno-linguistiques et culturelles se mobilisèrent également sur ce thème en 1990. Dans une configuration identitaire cristallisée pour les Magyars de Roumanie autour de la centralité de la langue, l'université « en hongrois » faisait figure d'université « hongroise ». Mais quelles furent les conditions politiques, institutionnelles et sociales de l'émergence accélérée de cette cause, au moment où un univers de représentations, de significations et de pratiques se redéployait selon un nouvel ordonnancement ? À la faveur de quel répertoire historique et symbolique et de quel travail sur ces matériaux des intellectuels et/ou de nouveaux acteurs politiques parvinrent-ils à construire la langue (de l'université, notamment) en indice du nouveau statut politique du groupe au sein de l'État roumain et, ainsi, à modeler un horizon d'avenir ? Quelles images sociales de l'identité ${ }^{5}$ ce projet d'université pour une langue traduisait-il, en les refaçonnant en même temps ?

2. Dans le domaine des humanités et des sciences, les universités de Bucarest, Iasi et Cluj sont en 1989 à la fois les plus anciennes et les plus prestigieuses. Durant l'année universitaire 19891990, on compte en Roumanie 44 établissements d'enseignement supérieur - tous publics, le secteur privé n'existant pas -, qui regroupent 11696 enseignants et 164507 étudiants. Parmi ces derniers, $58 \%$ suivent le cursus «du jour ", les autres étant inscrits en «formations du soir » ou «sans obligation de présence ». L’Université Babes-Bolyai regroupe 539 enseignants (dont 71 identifiés comme « Hongrois ») et 3010 étudiants (dont 661 «Hongrois »). Si elle est le seul établissement de la ville spécialisé en humanités et en sciences (ses diplômés étant en majorité destinés à une carrière d'enseignant de collège ou de lycée), d'autres établissements forment à Cluj des ingénieurs, des économistes, des professions médicales, des professions artistiques (peinture, musique). Cf. Veress K., « Magyar tagozatépítés a Babes-Bolyai Tudományegyetemen » [Départements hongrois à l'Université Babes-Bolyai], Romániai Magyar Évkönyv 2001, Temesvár, Kolozsvár, Polis Könyvkiadó, 2001, pp. 149-158 ; Sadlak J., "The Emergence of a Diversified System : the state/private predicament in transforming higher education in Romania", European Journal of Education, vol. 29, n¹, 1994, pp. 13-23.

3. Il s'agissait du bactériologiste roumain Victor Babes (1854-1926) et du mathématicien hongrois János Bolyai (1802-1860).

4. Déployés à plus de $90 \%$ en Transylvanie, les Hongrois, 1,6 millions selon les chiffres du recensement de 1992, représentaient 7,1\% de la population. En 2002, leur poids démographique déclinait à $6,6 \%$. Institutul national de statisticà, "Recensàmântul populatiei si locuintelor 2002 » [Le recensement de la population et des habitations 2002], Bucarest, 2002, http://www.insse.ro/cms/files/RPL2002INS/index_rpl2002.htm

5. Au sens où l'entendent Martina Avanza et Gilles Laferté dans leur lecture critique de plusieurs travaux consacrés à l'identité. Voir Avanza M., Laferté G., « Dépasser la "construction des identités" ? Identification, image sociale, appartenance ", Genèses, n 61, décembre 2005, pp. 134-152. 
L'articulation entre la formation historique de l'État, la construction de la nation et l'unification linguistique réalisée par des vecteurs institutionnels spécifiques (académie, université, école, notamment) mobilisés pour asseoir le pouvoir et le prestige d'une langue standardisée, a été étudiée dans de nombreux travaux consacrés au nationalisme et à sa diffusion ${ }^{6}$. Des sociologues, des socio-linguistes ou des anthropologues ont en outre envisagé les idéologies, les politiques et les pratiques linguistiques de manière non seulement à «dénaturaliser » la langue, à éclairer les liens entre les ordres sociaux et linguistiques, mais aussi à questionner les modalités de la domination de la langue d'État ${ }^{7}$. Car cette dernière fait autorité de manière différenciée parmi les groupes dits « minoritaires » selon leurs positions dans les imaginaires historiques et les hiérarchies symboliques, dans l'économie politique nationale et régionale ${ }^{8}$. Ces travaux ont ainsi articulé l'épaisseur socio-historique des pouvoirs de la langue et la contextualisation fine de leur redéploiement sur des échelles spatiales et temporelles imbriquées.

Dans cette perspective, la Roumanie et les conflits linguistiques au sortir du communisme fournissent un terrain d'observation riche. Les pratiques linguistiques y ont légitimé, par le passé, des revendications territoriales, la langue y étant promue depuis le XIXe siècle comme une synecdoque de cultures nationales territorialisées et un vecteur de concurrences élitaires hongroises et roumaines (et, dans une moindre mesure, allemandes). Le prisme spécifique adopté ici concerne les controverses autour de la (ré)ouverture de l'université publique de langue hongroise à Cluj-Napoca/Kolozsvár, telles qu'elles se déploient entre le moment de la chute de Nicolae Ceausescu et les premières élections « libres », législatives et présidentielles, du 20 mai 19909.

6. Voir notamment Anderson B., Imagined Communities: Reflections on the Origin and Spread of Nationalism, Londres, Verso, 1983 ; Hobsbawm E., Nations et nationalisme depuis 1780. Programme, mythe, réalité, Paris, Gallimard, 1992 ; Certeau M., Julia D., Revel J., Une politique de la langue. La Révolution française et les patois : L'enquête de Grégoire, Paris, Gallimard, 1975 ; Laitin D., Language repertoires and state construction in Africa, Cambridge, Cambridge University Press, 1992. Pour une historiographie centrée sur l'empire autrichien et l'Autriche-Hongrie voir Cohen G., Education and Middle-Class Society in Imperial Austria, 1848-1918, Purdue University Press, 1996 ; King J., Budweisers into Czechs and Germans. A Local History of Bohemian Politics, 1848-1948, Princeton et Oxford, Princeton University Press, 2002 ; Judson P.M., Guardians of the Nation. Activists on the Language Frontiers of Imperial Austria, Cambridge, London, Harvard University Press, 2006.

7. Bourdieu P., Ce que parler veut dire. L'économie des échanges linguistiques, Paris, Fayard, 1982 ; Langage et pouvoir symbolique, Paris, Seuil, 2001.

8. Gal S., "Contradictions of standard language in Europe: Implications for the study of practices and publics”, Social Anthropology, vol. 14, n² 2, 1987, pp. 637-653 ; Verdery K., "The Unmaking of an Ethnic Collectivity: Transylvania’s Germans”, American Ethnologist, vol. $12, \mathrm{n}^{\circ} 1,1985$, pp. 62-83.

9. Cette analyse repose sur le dépouillement des deux principaux quotidiens locaux, l'un de langue roumaine, Adevàrul în libertate [La vérité en liberté] devenu Adevàrul de Cluj [La Vérité de Cluj] et l'autre, de langue hongroise, Szabadság [Liberté], sur la période 22 décembre 1989 - 31 mai 1990. Des journaux et des mémoires d'acteurs politiques et universitaires engagés dans les luttes identitaires et les conflits linguistiques des années 1990 ont été également mobilisés, ainsi que les documents programmatiques de l'UDMR. Des entretiens ont été réalisés en 1996-1997 et en 2006-2007 dans le cadre d'enquêtes sur les politiques de l'iden- 
L'examen de ces controverses permet d'explorer les conditions et les modalités de cristallisation en une période singulière, celle du changement de régime, de répertoires discursifs et de catégories d'intelligibilité des enjeux de langue et d'université. Ces répertoires vont nourrir, au cours des deux décennies suivantes, des luttes identitaires hungaro-roumaines.

Au regard d'autres travaux croisant les enjeux d'université et de langues au XXe siècle, informés le plus souvent par la fréquentation de terrains occidentaux - notamment celui de l'Université catholique de Louvain, en Belgique, dont le dédoublement selon le clivage linguistique flamand/francophone en 1968 apparaît comme un épisode important dans la fédéralisation de l'État 10 - la configuration envisagée ici se singularise par son insertion dans des dynamiques de passage du communisme au postcommunisme, ainsi que par une trajectoire historique particulière. Cette dernière renvoie non seulement à des transformations socio-politiques intervenues au sein d'un État, elle est également le produit de concurrences entre deux États pour le contrôle d'un territoire. Dans un contexte d'incertitude politique et institutionnelle extrême, le temps court donne ainsi à voir le redéploiement d'idéologies linguistiques façonnées au fil des constructions stato-nationales rivales (hongroise et roumaine), nourries par des renversements des positions de domination à la suite de redéfinitions territoriales. Ces idéologies font également écho au travail d'égalisation relative des conditions sociales opéré en Roumanie par le régime communiste. Cette égalisation n'interdit toutefois pas les luttes de classement, notamment fondées sur la langue.

Le répertoire historique de la langue politisée de l'université est réactivé en décembre 1989 par des entrepreneurs identitaires qui se recrutent parmi des cadres d'université, des journalistes et des écrivains dotés de notoriété, des anciens diplômés de l'Université de Cluj, des cadres de la nomenklatura communistes marginalisés dans les années 1980. Ils mobilisent des capitaux et des ressources institutionnelles acquis sous le communisme pour s'avancer comme porteurs d'une "parole autorisée » exprimée au nom d'un groupe (hongrois de Roumanie ou roumain) ${ }^{11}$. Plusieurs d'entre eux vont investir le champ politique au cours de ces mois de bouleversement intense et de renouvellement des acteurs. L'incertitude caractérise également l'ordre universitaire ainsi que le fonctionnement de l'Université Babes-Bolyai, mis en conformité

tité. Enfin, nous avons eu recours à une littérature grise produite par des auteurs hongrois détenteurs de positions universitaires, en même temps experts et/ou conseillers associés à la définition des programmes de l'UDMR en matière d'enseignement supérieur. La reconstitution de la trajectoire historique de l'université repose sur les entretiens, le dépouillement des comptes-rendus des réunions préparatoires de la fusion des universités hongroise et roumaine et une littérature secondaire.

10. Laporte C., L'affaire de Lowvain (1960-1968), Paris, Bruxelles, De Boeck Université, 1999 ; De Coster M., Les enjeux des conflits linguistiques. Le Français à l'épreuve des modèles belge, suisse et canadien, Paris, L'Harmattan, 2007.

11. Bourdieu P., "Le mystère du ministère. Des volontés particulières à la "volonté générale" ", Actes de la recherche en sciences sociales, vol. 140, $\mathrm{n}^{\circ}$ 4, 2001, pp. 7-11, p. 10. 
idéologique sous le communisme, objet à la fin du régime de contestations multiples affectant, par delà la seule question linguistique, toutes les hiérarchies et les formes d'autorité. Enfin, un autre élément de spécificité du cas observé ici renvoie, dans une situation de recul démographique des Magyars en Roumanie, à de nouvelles circulations d'hommes, de biens et d'images qui relient la Roumanie à une Hongrie qui jouit au sortir du communisme d'un prestige international et d'une influence régionale accrus, ainsi que d'une séduction renouvelée au sein de la population dite minoritaire. Ces nouveaux flux ont pour effet d'intensifier la concurrence symbolique des deux langues sur un marché transylvain des biens symboliques désormais plus ouvert.

L'examen des luttes universitaires offre dès lors un éclairage sur l'éventail des significations que revêtent ces mobilisations linguistiques dans un temps court, accéléré, qui redéploie simultanément du temps moyen et long. L'horizon du possible en matière de redécoupage du monde social s'ouvre à la faveur d'une économie politique nationale et régionale bouleversée, ainsi que d'une réorganisation de l'ordre juridico-politique, mise en avant dans les travaux consacrés aux conflits (ethno)-linguistiques qui traversent, à la sortie du communisme, plusieurs sociétés est-européennes ${ }^{12}$. Des transformations sociales qui ne s'énoncent pas forcément dans les catégories de l'ethnicité entretiennent les mobilisations linguistiques, et ici, en l'occurrence, celles qui ont pour objet la mise en place d'une institution d'enseignement supérieur de langue hongroise qui s'adosse à une tradition.

\section{L'université, un emblème identitaire disputé}

Revendiquée dans les premières proclamations formulées par des personnalités issues des élites intellectuelles magyares, la réouverture de l'Université Bolyai de Cluj/Kolozsvár acquiert en l'espace de quelques semaines une saillance particulière, une pétition réunissant entre janvier et juin 1990178000 signatures. Cette résonance sociale est le fruit d'interactions qui renforcent la visibilité des catégories linguistiques dans l'interprétation des événements du présent. Les argumentaires hongrois croisent rapidement des contre-argumentaires roumains. Les uns et les autres établissent des continuités à partir de multiples discontinuités. La trajectoire de l’Université de Cluj et les luttes linguistiques qu'elle suscite éclairent en effet l'incorporation successive d'un territoire, la Transylvanie, dans deux États-nations, la Hongrie et la Roumanie, et les concurrences élitaires renforcées par l'instabilité des configurations étatiques et refaçonnées par les changements de l'ordre socio-politique ${ }^{13}$.

12. Voir notamment Csergö Zs., Talk of the nation. Language and conflict in Romania and Slovakia, Ithaca et Londres, Cornell University Press, 2007.

13. La principauté historique de Transylvanie avait appartenu au Royaume médiéval de Hongrie avant de connaître des tutelles impériales successives (ottomane, ensuite autrichienne). En 1867 elle est incorporée - avec l'accord des élites régionales s'identifiant majoritairement à la nation hongroise - à la composante magyare de l'empire austro-hongrois. A la fin de la première Guerre mondiale, avec d'autres territoires qui la bordent à l'ouest, elle est rattachée à la 
L'Université de Kolozsvár ouvre ses portes en 1872 et adopte en 1881 le nom de l'empereur d'Autriche et roi de Hongrie, François-Joseph. C'est le deuxième établissement d'enseignement supérieur dans ce pays et son rayonnement commence à s'étendre au-delà de son aire régionale à l'orée du XXe siècle. Les enseignements y sont dispensés quasi-exclusivement en hongrois, langue du pouvoir nobiliaire magyar et langue d’État envisagée comme un vecteur d'assimilation des populations non-hungarophones qui constituent $54,56 \%$ des habitants de la Hongrie en $1910{ }^{14}$. Seule une chaire de grammaire roumaine vient troubler ce monolinguisme ${ }^{15}$. La capitale de la Transylvanie résonne elle-même à l'époque des sonorités du hongrois, idiome des élites sociales et culturelles, les roumanophones - bilingues en majorité en milieu urbain - représentant en 1910 un peu plus de $15 \%$ des quelque 50000 habitants ${ }^{16}$.

La redéfinition de l'appartenance étatique de la Transylvanie à la fin de la Première Guerre mondiale bouleverse cette hiérarchie des groupes et de leurs langues. Pour l'État roumain dont le territoire agrandi comprend $28 \%$ de "minorités nationales », plus urbanisées que la population majoritaire, l'éducation secondaire et l'enseignement supérieur en roumain acquièrent une importance particulière, même si dans les villes transylvaines le hongrois reste très présent dans l'économie, les médias, la culture et les loisirs. Nationalisée, l'Université François-Joseph est rebaptisée Université de la Dacie supérieure Roi Ferdinand Ier, en hommage au souverain roumain. Le roumain, nouvelle langue d'État, y remplace le hongrois ${ }^{17}$. Licenciés pour avoir refusé de prêter serment à la Roumanie, les enseignants magyars émigrent pour la plupart en Hongrie. Par une astuce juridique, la fiction de la continuité institutionnelle de l'Université François-Joseph est toutefois maintenue : l'Université de Kolozsvár serait juste « déménagée » par l'État hongrois, qui continue à soutenir les institutions culturelles, les Eglises et le système scolaire confessionnel de la minorité magyare de Roumanie ${ }^{18}$. La nouvelle révision des frontières en cours entre 1940 et 1944 partage la Transylvanie entre les deux États. Se trou-

\footnotetext{
Roumanie au nom du poids démographique des Roumains (soit 53,8 \% d'habitants de langue maternelle roumaine en 1910). Les frontières entre la Hongrie et la Roumanie sont à nouveau redéfinies de 1940 à 1944, la Transylvanie étant partagée entre les deux États rivaux, alliés de l'Allemagne nazie. Le traité de paix de Paris de 1947 confirme l'appartenance de la Transylvanie réunifiée à l'État roumain. Pour une présentation synthétique, voir Brubaker R., Feischmidt M., Fox J., Grancea L., Nationalist Politics and Everyday Ethnicity in a Transylvanian Town, Princeton, Princeton University Press, 2006, pp. 56-88.

14. Cf. Gyáni G., Kövér Gy., Magyarország társadalomtörténete. A reformkortól a második világháborúig [L'histoire sociale de la Hongrie. De l'ère des réformes à la Seconde Guerre mondiale], Budapest, Osiris, 1998, p. 146.

15. Nastasà L., "University, Education and Culture in Kolozsvár/Cluj”, in Karády V., Nastasà L., The University of Kolozsvar/Cluj and the students of the Medical Faculty (1872-1918), Budapest, Cluj-Napoca, CEU Press, 2004, pp. 15-45

16. Brubaker R. et allii, op. cit., p. 93.

17. Livezeanu I., Cultural Politics in Greater Romania: Regionalism, Nation Building, and Ethnic Struggle, 1918-1930, Ithaca, Cornell University Press, 1995.

18. Bárdi N., "Hungary and the Hungarians Living Abroad: A Historical Outline", Regio, 2003, pp. 121-138.
} 
vant à nouveau sous souveraineté hongroise, Kolozsvár accueille désormais l'université « magyare » et voit s'éloigner l'université « roumaine », installée à Sibiu/Hermanstadt, centre urbain de la Transylvanie du sud sous contrôle roumain.

Par ses politiques d'ingénierie sociale - qui débouchent sur une réduction des disparités économiques à l'intérieur des groupes hongrois et roumain ainsi qu'entre ces derniers - et par ses innovations institutionnelles dans la gestion de la diversité ethno-linguistique inspirées en partie du modèle soviétique ${ }^{19}$, le régime communiste semble mettre un terme à cette compétition 20 . Rompant avec le nationalisme roumain de l' "époque bourgeoise », évinçant en même temps les élites historiques hongroises issues de la noblesse terrienne, des bourgeoisies et des Eglises catholiques et protestantes, l'État dont la haute administration comprend dans un premier temps de nombreux Magyars venus de la social-démocratie et du mouvement communiste, favorise l'émergence de nouvelles élites minoritaires formées en langue hongroise. Cette dernière est utilisée dans l'éducation, l'administration et la justice. Elle fait en outre fonction de langue officielle dans la "région autonome magyare/Mures » (1952-1968) située au centre de la Roumanie, à l'est de Cluj/ Kolozsvár, habitée par une population magyare-sicule 21 majoritaire $(77,32$ \%) à l'échelle du territoire qui a pour chef-lieu la ville de TârguMures/Marosvásárhely (77 \% de Hongrois). Cette langue hongroise promue après 1945 ne saurait toutefois se confondre avec celle du passé. Elle fait en effet l'objet d'un refaçonnage idéologique censé l'éloigner de l'imaginaire nationaliste, elle est requise pour fonder le nouvel ordre politique et social égalitaire.

L'ancienne capitale de la Transylvanie héberge quant à elle, à partir de 1946, deux universités d'État, de langue roumaine et hongroise, alors que Târgu-Mures/Marosvásárhely accueille un Institut de médecine et pharmacie et un Institut de théâtre, avec le magyar pour langue d'instruction. Ces différents établissements sont guidés par les mêmes valeurs, confrontés aux mêmes épurations des « ennemis de classe ». Les deux universités de Cluj assurent la formation en sciences humaines, sociales et « dures » et préparent des universitaires, des enseignants du secondaire, des journalistes ${ }^{22}$. Ce parallélisme ins-

19. Martin T., The affirmative action empire. Nations and nationalism in the Soviet Union, 19231939, Ithaca, Londres, Cornell University Press, 2001.

20. Bottoni S., "Reassessing the Communist Takeover in Romania. Violence, Institutional Continuity, and Ethnic Conflict Management”, East European Politics E Societies, vol. 24, $\mathrm{n}^{\circ} 1$, février 2010, pp. 59-89.

21. Installés dans les montagnes de la Transylvanie orientale, occupant initialement la fonction de garde-frontières du Royaume hongrois, les Sicules sont dotés de structures politiques et sociales propres jusqu'à la fin du XVIII ${ }^{\mathrm{e}}$ siècle, lorsque commence le processus d'identification à la nation politique et culturelle hongroise.

22. D'autres établissements d'enseignement supérieur de profil technique, médical, économique et artistique enrichissent l'offre universitaire et proposent quelques enseignements en hongrois, mais ne font pas l'objet de la même appropriation identitaire. 
titutionnel se prolonge jusqu'au printemps 1959 lorsque les autorités communistes y mettent un terme, tant sous l'effet de dynamiques politiques internes (consolidation de l'ordre communiste) que sous celui de dynamiques régionales plus larges (affaiblissement de la position de la Hongrie vis-à-vis de l'Union soviétique après la révolution de 1956, cette dernière ayant d'ailleurs suscité des mobilisations notamment dans les milieux universitaires hongrois de Cluj) ${ }^{23}$.

Plutôt saluée par les académiques roumains, la fusion des deux établissements est vécue sous le mode de la dépossession par les enseignants et étudiants hongrois, d'autant qu'elle est associée au suicide de trois cadres académiques, dont le dernier vice-président de l'Université Bolyai. L'institution de langue hongroise avait conforté l'évidence de l'appartenance magyare (fussent ses paramètres sociaux et sa marqueterie identitaire redéfinis) au moins autant, si ce n'est plus, qu'elle avait servi l'identification communiste. Les relevés des réunions qui se succèdent pour préparer la fusion attestent d'une interpénétration des idéologies nationaliste et communiste de la langue et d'un écart entre certaines visions roumaines et hongroises. Ainsi l'historien et archéologue roumain Constantin Daicoviciu (1898-1973), premier recteur de l'université après la fusion (il gardera cette fonction entre 1957 et 1968), est favorable à une hiérarchisation des deux langues au sein du futur établissement bilingue, le roumain devenant en tant que langue d'État, « la langue des universitaires et de la science de notre pays » ${ }^{24}$, le hongrois, subordonné, étant réservé à la formation des enseignants du secondaire. Les représentants de la hiérarchie universitaire hongroise, tout en regrettant le monolinguisme des étudiants magyars, défendent néanmoins un principe d'égalité des deux idiomes, car, selon la justification proposée par János Demeter, professeur de droit à l'Université Bolyai, «nous ne devons pas oublier que le peuple hongrois est conscient d'avoir une culture socialiste dans sa langue et qu'il chérit cette culture de langue hongroise $» 25$.

Sans complètement satisfaire la première orientation, l'unification entraîne toutefois un rétrécissement progressif de l'espace réservé au hongrois au sein de l'université bilingue Babes-Bolyai. Elle rend visible l'asymétrie des deux langues, dont l'une, langue d'État, peut articuler simultanément le registre du particulier (d'une identité roumaine) et de l'universel (de l'État) ${ }^{26}$. Le

23. Bottoni S., « Recepció és párhuzamosság. A romániai '56 és a magyar forradalom viszonya » [Réception et parallélisme. '56 en Roumanie et la révolution hongroise], Korunk, février 2006, disponible à l'adresse $:$ http://www.korunk.org/oldal.php ?ev =2006\&honap =2\&cikk =1529 [consultée le 11 juillet 2010]

24. « Compte-rendu de la réunion du 7 avril 1959 », in Andreescu A., Nastasà L., Varga A., (eds), Minoritàti etnoculturale. Màrturii documentare. Maghiarii din România (1956-1968) [Minorités ethnoculturelles. Témoignages documentaires. Les Magyars de Roumanie], Cluj, Centrul de Resurse pentru diversitate etnoculturalà, 2003, p. 449.

25. Ibid., p. 455.

26. Gal S., "Contradictions of standard language in Europe: Implications for the study of practices and publics", Social Anthropology, vol. 14, n², 2006, pp. 163-181, p. 168. 
nombre des enseignants et des étudiants magyars décline (entre 1959 et 1989, de 272 à 71 pour les premiers et de 1266 à 661 pour les seconds) et l'offre d'enseignements en hongrois se resserre. Pendant la même période, la population estudiantine de l'établissement - sélectionnée sur la base d'un examen d'admission après le baccalauréat - voit ses rangs diminuer pour passer de 4442 à 3007 27. Ces chiffres traduisent l'évolution plus générale de l'enseignement supérieur en Roumanie. Celui-ci avait connu une explosion du nombre des recrues et une diversification de leurs origines sociales par l'ouverture aux milieux ouvriers et paysans dans les années 1950, pour entamer dans les deux décennies suivantes une croissance plus modeste, concentrée surtout sur les profils technologiques aux dépens des sciences humaines et sociales, avant de connaître une forte contraction dans les années 1980. En 1989 la population estudiantine ne représente que 82,7 \% de celle de 1980 28. Vecteur de mobilité ascendante dans un premier temps, le diplôme universitaire devient dans la dernière décennie communiste un véhicule de reproduction sociale compte tenu du caractère très sélectif du recrutement, un bien de prestige rare et un critère de différenciation dans une société relativement égalitaire, dès lors qu'il fonctionne comme un pré-requis pour accéder à un statut supérieur.

Le renforcement de la présence du roumain dans l'enseignement supérieur 29 et le recul général des « humanités » entravent particulièrement la reproduction élitaire des Magyars qui pratiquent un bilinguisme asymétrique. Le poids des diplômés du supérieur - en 1956, de 1,3 \% parmi les habitants qui déclarent au recensement une appartenance roumaine contre $1 \%$ chez ceux qui définissent une appartenance hongroise, en 1992 de 5,3\% pour les premiers contre 3,6 \% pour les seconds 30 - en témoigne, même si les politiques linguistiques n'expliquent pas à elles seules ce décalage. L'enseignement secondaire transylvain connaît partiellement la même évolution, avec l'introduction de classes roumaines dans plusieurs anciens lycées hongrois dont certains sont en outre obligés d'abandonner un profil scientifique plus prestigieux pour adopter une orientation technologique. Le sentiment de déclassement s'adosse également à la marginalisation, dans les années 1970 et 1980, de la position du hongrois (et des cadres magyars) dans la haute nomenklatura et l'administration (la région autonome est supprimée en 1968), dans les médias, la culture et les sciences. L'industrialisation et l'urbanisation communistes contribuent par ailleurs à une modification de la démographie urbaine marquée par la « roumanisation » des villes transylvaines ${ }^{31}$.

27. Veress K., « Magyar tagozatépítés a Babes-Bolyai Tudományegyetemen » [Départements hongrois à l'Université Babes-Bolyai], Romániai Magyar Évkönyv 2001, Temesvár, Kolozsvár, Polis Könyvkiadó, 2001, pp. 149-158.

28. Rotariu T., Dan M., Mezei E.,Veres E., « Invàtàmântul superior între inertie si schimbare » [L'enseignement supérieur entre inertie et changement], Revista de cercetàri sociale, $\mathrm{n}^{\circ} 1$, mars 1995, pp. 51-64.

29. Les bacheliers hongrois peuvent néanmoins passer les concours d'admission à l'université dans leur langue maternelle avant de poursuivre les études en roumain.

30. Veres V., "Social Stratification and Ethnicity in Transylvania. How does social class matter?", International Journal of Sociology, vol. 36, $\mathrm{n}^{\circ}$ 1, printemps 2006, pp. 28-45, p. 29. 
Ces changements entretiennent des rêves d'ailleurs que l'on identifie progressivement à partir des années 1970 à la Hongrie. Les migrations dirigées vers cette destination touchent fortement les diplômés hongrois du supérieur. Sans être réservées exclusivement aux Magyars, car des Roumains désireux de quitter un pays plongé dans le marasme économique et subissant un renforcement du contrôle idéologique s'engagent également dans cette voie, elles s'accélèrent à partir de 1987 avec quelques 15000 départs par an ${ }^{32}$. Ce choix reflète le décalage de plus en plus marqué entre une Hongrie perçue comme la «baraque la plus gaie du camp communiste », où l'État, divers groupes politiques et intellectuels et les médias, investissent à nouveaux la cause des minorités magyares, tissent des liens transfrontaliers et parcourent à nouveau la Transylvanie 33 et une Roumanie isolée sur le plan international, qui allie nationalisme et raidissement idéologique communiste. Avec cet horizon hongrois comme promesse de salut, la valeur sociale et affective du magyar est rehaussée à la fin de la période communiste. Ces mutations ravivent des imaginaires de la supériorité magyare adossée à une langue, imaginaires réactualisés malgré l'égalisation des conditions sociales. Elles refaçonnent le bilinguisme asymétrique des Magyars et alimentent les mobilisations postcommunistes.

\section{9 au miroir de la langue politisée (de l’université)}

La chute du communisme, et, dans son sillage, la reconfiguration des arènes du pouvoir, fournissent des opportunités politiques et discursives pour investir le répertoire historique de la langue politisée et en particulier de la langue politisée de l'éducation. Néanmoins, l'appartenance ethno-linguistique n'apparaît pas, en décembre 1989, dans la phase la plus intense des mobilisations anticommunistes réprimées initialement, comme un facteur de clivage ${ }^{34}$. Les foules bigarrées qui remplissent les centres des villes transylvaines à l'annonce de la fuite du chef de l'État Nicolae Ceausescu, le 22 décembre 1989, brandissent le même drapeau roumain privé de ses armoiries communistes et entonnent les mêmes chants. Ici et là, des orateurs improvisés alternent roumain et hongrois au nom d'une nouvelle fraternité formulée en termes transethniques ${ }^{35}$. Progressivement, l'évènement nourrit néanmoins des construc-

31. Si le recul démographique des Hongrois est resté mesuré à l’échelle de la Transylvanie (25\% en 1948, $20 \%$ en 1992), leur poids dans la population urbaine de la province passe de 31,60\% en 1956 à 20,30\% en 1992. Pour le nationalisme roumain des années 1970-1980 notamment dans les milieux intellectuels, voir Verdery K., National Ideology under Socialism, Berkeley, University of California Press, 1991.

32. Horváth I., «A migráció hatása a népesség elöszámítására » [Les effets des migrations sur le décompte de la population], Magyar Kisebbség, vol. 7, $\mathrm{n}^{\circ} 4,2002$, disponible à l'adresse : http://www.hhrf.org/magyarkisebbseg/0204/m020403.html [consultée le 8 juillet 2010]

33. Kürti L., The Remote Borderland: Transylvania in the Hungarian Imagination, New York, State University of New York Press, 2001 ; Culic I., "Dilemmas of Belonging: Hungarians from Romania”, Nationalities Papers, vol. 34, n²2, mai 2006, pp. 175-200.

34. Siani-Davies P., The Romania Revolution of December 1989, Ithaca, NY, Cornell University Press, 2005. 
tions plurielles de l'avenir, partiellement façonnées par des représentations et des perceptions du passé ${ }^{36}$. La langue et ses déclinaisons dans les institutions de l'éducation qui avaient en partie modelé l'ordre du prestige à l'époque communiste en viennent à constituer une cause et en même temps un vecteur des mobilisations.

Fondée par quelques intellectuels hongrois de Bucarest réunis autour de l'écrivain Géza Domokos (1928-2007) 37, l’Union démocratique des Magyars de Roumanie (UDMR) est présente dans l'espace public dès le 25 décembre 1989 et est légalisée un mois plus tard ${ }^{38}$. Elle se donne pour but de « représenter et de défendre les intérêts magyars et d'accueillir tout mouvement, organisation ou association, qui accepte ses principes fondamentaux 39 ». D'autres initiatives qui adhèrent au groupe de Bucarest se cristallisent sous la même bannière ethno-linguistique dans plusieurs villes de Transylvanie, et notamment à Cluj/Kolozsvár (autour essentiellement d'universitaires) 40 et TârguMures/Marosvásárhely. En peu de temps, l’Union parvient à se doter de représentations locales, municipales et départementales maillant la Transylvanie où résident $99 \%$ des Magyars. Elle ouvre son parapluie sur une myriade d'organisations et d'associations de langue hongroise qui essaiment sur des bases idéologiques, confessionnelles, générationnelles ou professionnelles. Dans un esprit de coopération transethnique supposé indiquer la rupture avec la période communiste, elle est associée aux nouvelles structures provisoires du pouvoir constituées à Bucarest autour du Front du salut national (FSN). D'anciens hauts cadres communistes marginalisés et des membres de l'intelligentsia magyare accèdent pendant les mois qui séparent les mobili-

35. Brubaker R. et allii, Nationalist Politics and Everyday Ethnicity in a Transylvanian Town, op. cit., pp. 119-122 ; Goina C., The ethnicization of politics : the case of Târgu-Mures, 1990, MA Thesis, Départment de Sociologie, Université de Californie, Los Angeles.

36. Farge A., "Penser et définir l'événement en histoire », Terrain, Qu'est-ce qu'un événement ?, $\mathrm{n}^{\circ} 38$, mars 2002, pp. 69-78.

37. G. Domokos avait entamé sa formation universitaire à l'Université Bolyai avant de la poursuivre à Moscou, à l'Institut Maxim Gorki. Depuis 1969, il dirige la très prestigieuse maison d'édition « Kriterion ", qui publie des ouvrages dans les langues des minorités, essentiellement en hongrois. Jusqu'en 1984 il fut également membre suppléant du Comité central du Parti communiste.

38. Capelle-Pogàcean A. et Ragaru N., «En quoi les "partis ethniques” sont-ils "ethniques” ? Les trajectoires du MDL en Bulgarie et de l'UDMR en Roumanie ", Questions de Recherche / Research in Question, $\mathrm{n}^{\circ}$ 25, juin 2008, http://www.ceri-sciences-po.org/ publica/question/qdr25.pdf

39. "A Romániai Magyar Demokrata Szövetség szándéknyilatkozata " [Déclaration d’intention de l'UDMR], in Bárdi N., Eger Gy. (eds.), Utkeresés és integráció. Válogatás a határon tuli magyar érdekvédelmi szervezetek dokumentumaiból 1989-1999, Budapest, Fondation Teleki László, 2000, p. 53.

40. Les quinze signataires de « Hivó szó » [Appel], publié dans le quotidien local de langue hongroise Szabadság dès le 24 décembre 1989 sont universitaires à Babes-Bolyai et pour certains anciens hauts cadres de l'Université Bolyai (notamment un ancien vice-recteur). Ils sont historiens, philosophes, linguistes, spécialistes de la littérature hongroise, animateurs d'une des principales revues littéraires de langue hongroise. Plusieurs d'entre eux ont été éloignés de l'université en raison des prises de position critiques à l'égard du régime, dans les années 1980 ou ont connu la prison politique dans les années 1950. Voir « Hivó szó » [Appel], Szabadság, 24 décembre 1989, p. 1. 
sations anticommunistes de décembre 1989 et les premières élections législatives et présidentielles du 20 mai 1990, à des fonctions importantes dans les ministères de l'Éducation et de la Culture (vice-ministres) et dans les conseils départementaux et municipaux du FSN en Transylvanie.

Dans ces nouvelles organisations magyares, les diplômés de l'enseignement supérieur, en particulier des «humanités », passés dès lors par l’Université Bolyai ou Babes-Bolyai - principal établissement assurant une formation en hongrois - sont sur-représentés. Leur parole fait autorité pas seulement en raison de savoir-faire spécifiques dans le maniement de la langue. Elle fait écho à un messianisme de l'intelligentsia magyare en situation minoritaire, à un rôle construit en situation de fragilisation des anciennes élites politiques et sociales après l'incorporation de la Transylvanie à la Roumanie en 1918, un rôle encore renforcé par les transformations sociales et politiques de la période communiste. Ce lacis de réseaux dont la formation rapide s'appuie ainsi sur des imaginaires historiques et des ressources institutionnelles préexistantes, projette l'image d'une communauté hongroise qui a la langue pour principal support d'identification, celle-ci marquant en même temps la frontière la séparant des Roumains. Les controverses autour de l'Université Bolyai prolongent jusqu'à un certain point un débat plus ample sur la langue de l'éducation en Transylvanie ${ }^{41}$, tout en participant à la redéfinition des représentations identitaires magyares. Exiger le rétablissement de l'Université Bolyai permet de donner sens à la césure de 1989. Celle-ci doit ouvrir à un nouvel ordre politique où l'éducation en langue maternelle à tous les niveaux serait juridiquement et institutionnellement garantie. Elle est supposée clore en même temps un passé dont il convient de redresser les injustices. Leurs victimes ne sont pas désignées au sein des catégories supérieures et moyennes affaiblies par le tournant nationaliste du régime communiste roumain et par les migrations successives vers la Hongrie qui ont scandé le $\mathrm{XX}^{\mathrm{e}}$ siècle ${ }^{42}$. C'est la communauté ethno-linguistique qui est désignée comme ayant subi collectivement la violence symbolique de la disparition de l'Université Bolyai et les tentatives d' « assimilation forcée ».

Evoquée dans les premiers textes programmatiques de décembre 1989 qui ébauchent à Bucarest ou à Cluj/Kolozsvár, autour d'académiques, la nouvelle relation entre l'État roumain et le groupe hongrois, l'Université Bolyai apparaît en pièce maîtresse d'un réseau d'établissements d'éducation supérieure de langue maternelle qui prolonge un système autonome d'instruction secondaire ${ }^{43}$. Les acteurs magyars - qu'ils soient en train d'investir le champ politique comme le nouveau président de l'UDMR, G.Domokos, ou qu'ils adoptent un profil d'expert - n'envisagent pas la possibilité de créer une université privée alors que plusieurs établissements de ce genre sont fondés en Roumanie

41. «Màsuri drepte în învàtàmînt » [Des mesures justes dans l'enseignement], Adevàrul în libertate, 26 janvier 1990, p. 2. 
en 1990 44. La dimension «publique » de l'Université de langue hongroise se décline dans le registre de la citoyenneté. Cette revendication vis-à-vis de l'État se justifie tant par l'épaisseur historique de la présence de la communauté sur le territoire de la patrie transylvaine, que par sa démographie. La sous-représentation des Magyars parmi les diplômés du supérieur fournit un autre argument mobilisé dans la démarche de persuasion.

Le 14 janvier 1990, le quotidien roumain de Cluj/Kolozsvár rend publique une déclaration appartenant à d' " anciens enseignants de l'Université Bolyai et actuels professeurs d'appartenance hongroise de l'Université BabesBolyai » qui définit ces cadres d'interprétation de la cause. La réouverture de l'université est défendue au nom d'une trajectoire institutionnelle de longue durée injustement brisée et de "l'égalité et la fraternité [fondées] non seulement sur des bases individuelles, mais aussi comme une relation entre deux institutions autonomes 45 ». Cette prise de position surprend à ce moment les cadres roumains de l'Université de Cluj. Le rejet partagé du communisme n'interdit pas la différenciation des régimes d'expérience de celui-ci, ni les asymétries. Le vécu roumain est moins (ou autrement) marqué par la dimension anti-magyare du communisme tardif. Ce, d'autant que le clivage linguistique nourri au niveau institutionnel par l'éviction progressive du hongrois, n'avait toutefois pas infiltré d'une manière également intense l'ensemble des relations sociales. Des coopérations professionnelles, des relations de clientèle, des pratiques d'entraide et de bon voisinage et des amitiés s'étaient poursuivies nonobstant les accents anti-hongrois - eux-mêmes euphémisés - de la rhétorique politique officielle. En outre, tous les enseignants de l'Université BabesBolyai - formant un corps académique profondément renouvelé dans les années 1960 - n'avaient pas connu l'époque du parallélisme institutionnel. Ceux d'entre eux qui l'avaient expérimenté n'en cultivaient pas, quant à eux, le même souvenir que les universitaires hongrois.

42. Entre 1918 et 1922, 197000 Magyars, grands propriétaires terriens, militaires, fonctionnaires, personnel enseignant quittent la Transylvanie. Cf. Livezeanu I., Cultural Politics in Greater Romania, op. cit., p. 137. Certains d'entre eux reviennent entre 1940 et 1944, mais la fin de la Seconde Guerre mondiale s'accompagne de nouveaux déplacements, avec quelque 130000 Hongrois qui prennent le chemin de la Hongrie ou de l'Occident. Tóth P.P., « Adalékok a magyar migrációs politikai stratégia kialakításához », Kisebbségkutatás, vol. 8, n 3, 1999, http://www.hhrf.org/kisebbsegkutatas/kk_1999_03/cikk.php ?id =110 [consultée le 8 juillet 2010].

43. "Cuvinte pentru început » [Paroles pour un nouveau commencement], Adevàrul în libertate, 23 décembre 1989 ; "A Romániai Magyar Demokrata Szövetség szándéknyilatkozata », op. cit., p. 57.

44. Sadlak J., "The Emergence of a Diversified System: the state/private predicament in transforming higher education in Romania”, European Journal of Education, vol. 29, $\mathrm{n}^{\circ}$. 1, 1994, pp. 13-23 ; Eisemon T.O., Mihàilescu I., Vlàsceanu L., Zamfir C., Sheehan J., Davis Ch.H., "Higher Education Reform in Romania", Higher Education, vol. 30, n² 2, septembre 1995, pp. 135-152.

45. «Declaratie » [Déclaration], Adevàrul în libertate, 14 janvier 1990, p. 2. 
Dès lors, pour de nombreux cadres roumains de l'université, le clivage linguistique ne fournit pas d'indice pertinent pour évaluer l'expérience communiste et penser le postcommunisme, au moins pas dans le domaine de l'enseignement supérieur. Les souffrances et les privations ont pu acquérir des notes spécifiques dans les milieux de langue hongroise, sans être fondamentalement différentes de celles éprouvées par la majorité. Des voix « roumaines » s'expriment dès lors, s'identifiant comme « universitaires roumains » qui répondent aux «collègues magyars » : "Quelle est donc cette absence de droits que vous évoquez alors que jusqu'à la fin de la période communiste vous avez eu des cours et des travaux pratiques dans votre langue maternelle et vous avez joui $\mathrm{du}$ respect de vos collègues roumains ? » « Nous avons souffert ensemble 46 », voici le constat opposé à la requête magyare.

Autour de l'Université de Cluj/Kolozsvár, ancienne ville de rayonnement de la noblesse hongroise, désormais « roumaine » car le poids des Magyars y dépasse de peu les $22 \%{ }^{47}$, se cristallisent ainsi, dès les premières semaines de la période postcommuniste, deux lectures initialement asymétriques et ensuite divergentes du passé qui participent à la politisation de la langue. La « réouverture » de l'université magyare est rejetée comme "séparatisme » par des enseignants roumains. Une rhétorique nationaliste se cristallise dans ce groupe, impulsée notamment par des cadres qui avaient traversé l'épisode 1940-1944 et la migration forcée de l'université roumaine de Cluj à Sibiu. Ces cadres récusent la légitimité d'une revendication de continuité avec une institution «stalinienne » qui fut imposée de l'extérieur (par l'Union soviétique) 48. Ils réactualisent le souvenir de la division du territoire transylvain entre 1940 et 1944. Le clivage linguistique cristallisé autour de l'université en janvier 1990 fait dès lors écho à d'anciennes rivalités pour le contrôle des institutions d'éducation, devenues à l'âge de l'État-nation des supports d'imposition d'une langue d'État et des vecteurs de prestige, de pouvoir et de contrôle du territoire transylvain. La scission de l'Université de Cluj, comme celle d'autres établissements d'instruction secondaire de Transylvanie revendiquée dans plusieurs villes - qui bénéficient alors de l'appui d'acteurs politiques de Hongrie, entrés eux-mêmes dans la première campagne électorale du postcommunisme, visibles également en Roumanie - apparaît dans cette interprétation comme le prélude d'une partition territoriale.

46. «Punctul nostru de vedere. Ràspuns colegilor maghiari » [Notre point de vue. Une réponse à nos collègues magyars], Adevàrul în libertate, 24 janvier 1990, p. 2 ; «Separatism sau armonie ? " [Séparatisme ou harmonie ?], Adevàrul în libertate, 27 janvier 1990, p. 2 ; «Incotro, universitatea clujeanà ? " [Dans quelle direction va l'Université de Cluj ?], Adevàrul în libertate, 15 février 1990, p. 1.

47. Le poids des locuteurs de roumain passe de 48,2 \% en 1956 à 75,6 \% en 1992. Brubaker R. et allii, op. cit., p. 93.

48. Voir notamment l'argumentaire du philosophe Dumitru D. Rosca (1895-1980). Prof.Dr. Dumitru D. Rosca, "Pledoarie împotriva separàrii » [Plaidoyer contre la séparation], Adevàrul de Cluj, 28 janvier 1990, p. 2. 
Progressivement, les controverses autour de la langue de l'éducation qui se déploient à un rythme soutenu embrassent ainsi non seulement la durée du communisme, mais la temporalité plus ample des constructions stato-nationales. L'université de langue hongroise Bolyai devient, pour les entrepreneurs magyars, le symbole d'un ancien prestige et d'une dépossession tragique dont la fin de la Première Guerre mondiale marque le début. Pour des académiques roumains, elle n'est qu'une illustration parmi d'autres des privilèges dont auraient joui, aux dépens de la population roumaine majoritaire en Transylvanie, les dominants hongrois, et ce tant à l'époque austro-hongroise qu'à l'époque du communisme stalinien internationaliste de la fin des années 1940 et des années 1950 avant que le régime ne s’ « autochtonise ».

\section{Mobilisations réticulaires : faire communauté}

Les disputes autour de la réouverture de l'Université Bolyai échappent au cercle étroit des initiés universitaires à la faveur de mobilisations où se croisent des logiques locales, nationales et régionales. La cause de l'université de langue hongroise est promue dans le cadre organisationnel fourni par l'UDMR. Elle est propagée par une presse locale et nationale de langue hongroise qui avait survécu aux années 1980 et augmente rapidement son nombre de pages, ainsi que par des médias de Hongrie, radios et télévision en particulier, dont certains peuvent être captés sur le territoire transylvain. La diffusion du thème de l'université est en même temps facilitée par la multipositionnalité des acteurs magyars - universitaires/experts/hommes politiques - et l'enchevêtrement de champs politiques et sociaux. Parfois, les mêmes individualités apparaissent dans les instances nationales de l'Union et dans les différentes associations de langue hongroise. Plusieurs universitaires de Cluj font temporairement l'expérience des administrations centrales. Fondée le 17 mars 1990 à Cluj, l'association Bolyai qui regroupe de nombreux académiques magyars et s’impose dans les années 1990 comme le principal acteur collectif chargé de développer l'enseignement supérieur en langue maternelle en Transylvanie et de préparer la réouverture de l'université hongroise, éclaire cet enchâssement des domaines. Certains de ses initiateurs sont présents dans les structures dirigeantes de l'UDMR. D'autres viennent de l'Union nationale des étudiants magyars de Roumanie, assurant ainsi un relais vers cet espace ${ }^{49}$. Enfin, l'association Bolyai tisse des liens institutionnels avec l'État hongrois, notamment en supervisant la distribution des différentes aides consacrées par celui-ci à la formation des élites hongroises de Roumanie, initialement des bourses d'études offertes aux candidats transylvains qui souhaitent poursuivre leurs études supérieures dans les universités de Hongrie, soit annuellement quelque 3000 jeunes magyars au début des années 199050.

49. Udvardy F., «A romániai magyar kisebbség történeti kronológiája 1990-2003 » [Chronologie historique de la minorité magyare de Roumanie], http://udvardy.adatbank.transindex.ro/ [consultée le 18 juin 2010]. 
La résonance sociale de la cause de l'Université Bolyai tient également à la politisation de la population étudiante. Cet engagement n'est pas spécifique aux Hongrois. Si à Bucarest et dans les grandes villes universitaires de Roumanie, les étudiants réunis dans de nouveaux syndicats organisent dès janvier 1990 des grèves et des manifestations de rue pour exiger la réforme de l'université et des conditions d'admission dans les établissements d'enseignement supérieur, à Cluj, leurs camarades rassemblés dans des organisations de langue hongroise avancent, outre ces demandes «ethniquement neutres », des revendications propres. A l'Université Babes-Bolyai, une grève est lancée le 12 mars 1990 avec pour mot d'ordre non seulement le rétablissement de l'université magyare, mais aussi l'introduction de la parité linguistique dans les conseils académiques et la mise en place de quotas linguistiques à l'admission à l'université ${ }^{51}$. Les porte-parole des étudiants roumains rejettent pour leur part, compte tenu de la rareté des places, le recours aux catégories ethno-linguistiques pour réguler l'admission. Ils mettent en avant le principe du mérite qui seul doit prévaloir dans le monde académique. Le clivage ethno-linguistique se prolonge dès lors dans l'espace étudiant de l'Université Babes-Bolyai, nonobstant la réitération de la volonté de dialogue et les rapprochements opérés dans le cadre de manifestations de rue qui interrogent selon un mode «transethnique » l'évolution politique à Bucarest au nom de la « révolution (anti-communiste) confisquée » par les cadres issus de la nomenklatura ${ }^{52}$.

Enfin, la visibilité de la cause de l'Université Bolyai reflète également le travail de coordination symbolique assuré par les différentes organisations magyares, l'UDMR en tête, qui font le lien entre des actions collectives ancrées dans des sites locaux ${ }^{53}$. L'on observe ainsi des mimétismes et des mises en écho entre les mobilisations lycéennes et étudiantes à Cluj/Kolozsvár et celles, plus massives, déployées à Târgu-Mures/Marosvásárhely dès le mois de février 1990. Dans cet ancien centre de la Région autonome magyare où les Hongrois ne dépassent que de peu les $50 \%$, alors qu'ils représentaient $77 \%$ dans les années 1950 54, l'ethnicisation de la scène politique locale se prolonge dans la vie quotidienne. Les disputes autour d'un vieux lycée qu'il s'agit de reconvertir à son monolinguisme magyar traditionnel et de l'Institut de médecine et de pharmacie qui avait vu le renversement spectaculaire des proportions ethniques aux dépens du corps académique hongrois, y nourrissent un cycle de mobilisations et contre-mobilisations. Les 19 et 20 mars 1990, celui-

50. Bárdi N., Berki A., Ulicsák Sz., Erdélyi Magyar Tudományegyetem megvalósíthatósági tanulmánya [Etude de faisabilité de l'université magyare des Sciences de Transylvanie], Budapest, HTMH, 2001.

51. "Grevà la Universitate " [Grève à l'université], Adevàrul de Cluj, 17 mars 1990, p. 2.

52. «Dorinta de dialog » [Le volonté de dialogue], Adevàrul de Cluj, 18 mars 1990, p. 1.

53. Stroschein S., "Microdynamics of Bilateral Ethnic Mobilization", Ethnopolitics, vol. 10, n 1, 2011, pp. 1-34.

54. Le poids de la population roumaine y passe de $22,41 \%$ en 1956 à $46,70 \%$ en 1992. Cf. Varga A., « Erdély etnikai és felekezeti statisztikája (1850-1992) » [La statistique ethnique et confessionnelle de la Transylvanie (1850-1992)], disponible à l'adresse : http://varga.adatbank.transindex.ro/ [consultée le 13 juillet 2010]. 
ci débouche sur des affrontements intercommunautaires qui font dans cette ville six morts et quelques centaines de blessés 55 .

Les demandes linguistiques magyares rencontrent en effet des mobilisations roumaines, menées par des acteurs issus non seulement des cercles universitaires et des milieux enseignants, mais aussi des milieux de l'armée, des renseignements, de la justice, de la santé, soit un personnel roumain dont la mobilité sociale fut favorisée dans les villes transylvaines par l'orientation nationaliste de l'État communiste. Or, le nouveau déploiement du hongrois dans le domaine de l'éducation est perçu dans ce contexte régional comme une menace. Le 6 février 1990, une organisation nationaliste, l'Atre roumain, voit ainsi le jour à Târgu-Mures. De ce terreau émerge le 15 mars 1990, quelques semaines avant les premières élections législatives et présidentielles, une nouvelle formation politique, le Parti de l'union nationale des Roumains de Transylvanie, rebaptisé quelques mois plus tard Parti de l'unité nationale roumaine (PUNR). Ses représentants occuperont différentes positions gouvernementales -notamment dans le domaine de l'éducation - jusqu'en 1996. En outre, le PUNR contrôlera la mairie de Cluj-Napoca de 1992 à 2004 56. Les anciennes rivalités élitaires connaîtront désormais des traductions partisanes qui consolideront le clivage ethno-linguistique sur la nouvelle scène politique ${ }^{57}$.

Dans ce contexte, la cause de l'université d'État de langue hongroise restera au programme de l'UDMR explicitement jusqu'en 1995, d'une manière plus euphémisée par la suite ${ }^{58}$, sans néanmoins être satisfaite. Sa présence fait sens en rapport avec Fune définition spécifique du groupe magyar de Roumanie. Ce dernier n'est pas saisi dans les années 1990 à travers la catégorie de «minorité », mais par le biais de celle de «communauté autonome », formant une «société complète », riche notamment d'élites dont la reproduction requiert une instruction universitaire en langue maternelle dans des établissements qui se trouveraient sous contrôle communautaire.

55. “News From Romania: Ethnic Conflict in Targu-Mures”, News from Helsinky Watch, May 1990, p. $1-8$.

56. Gallagher T., Democratie si nationalism în România [Démocratie et nationalisme en Roumanie], Bucarest, All Educational, 1998, p. 114 ; Mungiu-Pippidi, A., Transilvania subiectivà [La Transylvanie subjective], Bucarest, Humanitas, 1999.

57. Pour plus de détails sur le déploiement du clivage ethno-linguistique sur la scène politique roumaine post-communiste voir Capelle-Pogàcean A. et Ragaru N., "En quoi les "partis ethniques” sont-ils “ethniques” ? Les trajectoires du MDL en Bulgarie et de l'UDMR en Roumanie ", op. cit. ; Csergö Zs., "Beyond Ethnic Division: Majority-Minority Debate About the Postcommunist State in Romania and Slovakia”, East European Politics and Societies, vol. 16, n ${ }^{\circ} 1,2002$, p. 1-29.

58. Egyed P., «Az autonómia, az egyetem és a család kérdése az RMDSZ-programokban », Magyar Kisebbség, vol. 6, $\mathrm{n}^{\circ}$ 1, 2000, http://www.jakabffy.ro/magyarkisebbseg/ index.php ?action $=$ cimek\&lapid $=14 \&$ cikk $=$ m000118.html 


\section{Conclusion}

Les engagements autour de la langue de l'enseignement supérieur participent à la fois d'une économie locale et régionale de rivalités retravaillées par l'ordre communiste - dont témoigne la « roumanisation » des villes transylvaine - et de logiques politiques nationales nourries par la question de la place du groupe hongrois dans l'État postcommuniste. La reconfiguration des positions internationales respectives de la Hongrie et de la Roumanie à partir des années 1970, accélérée durant la décennie qui précède la sortie du communisme, renforce des perceptions asymétriques de la place du clivage linguistique instauré entre le roumain, langue d'État et le hongrois, langue d'une minorité en Roumanie. Un écart s'établit entre les usages institutionnels de l'idiome minoritaire et ses usages sociaux. La reproduction sociale des élites magyares transylvaines est entravée par le rétrécissement de la place du hongrois dans un système d'enseignement supérieur qui fournit l'un des rares signes de statut social dans une société qui se prétend égalitaire et qui connaît néanmoins un processus de différenciation. L’imaginaire du dépérissement magyar en Transylvanie qui avait émergé après 1918 est réactualisé.

Dans le nouveau contexte politique qui s'esquisse après la chute du régime communiste, les entrepreneurs politiques hongrois issus des élites intellectuelles produites par le communisme et d'une nomenklatura marginalisée dans les années 1980 approchent la langue (et la langue de l'éducation) comme un cadre et un vecteur des mobilisations. Celle-ci est appelée à façonner un champ culturel partagé, à assurer la transmission d'une mémoire collective et à s'incarner dans des institutions communautaires (écoles et université au premier plan), lieux de socialisation, de reproduction de l'ethnicité et d'images sociales du groupe, qu'il s'agit de faire renaître ou de consolider. Elle joue aussi une fonction de représentation, sa visibilité publique étant la mesure de la reconnaissance du groupe hongrois par l'État roumain. Enfin, elle est valorisée en tant que lien avec la Hongrie voisine, «État-parent » perçu comme plus prospère et plus occidentalisé, qui soutient politiquement, symboliquement et financièrement les différentes organisations de langue magyare. La résonance sociale de la cause d'une université en hongrois et pour les Hongrois tient à ces déclinaisons multiples. 